PROCEEDINGS OF THE

AMERICAN MATHEMATICAL SOCIETY

Volume 133, Number 5, Pages 1365-1369

S 0002-9939(04)07647-6

Article electronically published on October 18, 2004

\title{
EXISTENCE OF SOLUTIONS FOR THREE-POINT BOUNDARY VALUE PROBLEMS FOR SECOND ORDER EQUATIONS
}

\author{
JOHNNY HENDERSON, BASANT KARNA, AND CHRISTOPHER C. TISDELL \\ (Communicated by Carmen C. Chicone)
}

\begin{abstract}
Shooting methods are employed to obtain solutions of the threepoint boundary value problem for the second order equation, $y^{\prime \prime}=f\left(x, y, y^{\prime}\right)$, $y\left(x_{1}\right)=y_{1}, y\left(x_{3}\right)-y\left(x_{2}\right)=y_{2}$, where $f:(a, b) \times \mathbb{R}^{2} \rightarrow \mathbb{R}$ is continuous, $a<x_{1}<x_{2}<x_{3}<b$, and $y_{1}, y_{2} \in \mathbb{R}$, and conditions are imposed implying that solutions of such problems are unique, when they exist.
\end{abstract}

\section{INTRODUCTION}

In this paper, we are concerned with the existence of solutions for three-point boundary value problems for the second order ordinary differential equation

$$
y^{\prime \prime}=f\left(x, y, y^{\prime}\right), \quad a<x<b .
$$

In particular, given points $a<x_{1}<x_{2}<x_{3}<b$, and $y_{1}, y_{2} \in \mathbb{R}$, we shall consider, in some sense, a uniqueness implies existence result for solutions of (1) satisfying the boundary conditions

$$
y\left(x_{1}\right)=y_{1}, \quad y\left(x_{3}\right)-y\left(x_{2}\right)=y_{2} .
$$

We assume throughout the following:

(A) $f:(a, b) \times \mathbb{R}^{2} \rightarrow \mathbb{R}$ is continuous.

(B) Solutions of initial value problems for (1) are unique and exist on all of $(a, b)$.

Interest in multipoint boundary value problems for second order differential equations has been ongoing for several years, with much of the attention given to positive solutions. For a small sample of such work, we refer the reader to works by Bai and Fang [1, Gupta and Trofimchuk [5, Ma [15, 16] and Yang [20].

This question of uniqueness implying existence for solutions of boundary value problems enjoys quite a history for ordinary differential equations as well as finite difference equations, and recently such questions have been addressed for dynamic equations on time scales. For ordinary differential equations, the papers by Hartman [6], Klaasen [13, Lasota and Luczynski [14, Henderson [7, Jackson and Schrader [12], and Peterson [17] are significant contributions, and for finite difference equations, the papers by Davis and Henderson [4] and Henderson [8, 9]

Received by the editors October 30, 2003 and, in revised form, December 30, 2003.

2000 Mathematics Subject Classification. Primary 34B15; Secondary 34B10.

Key words and phrases. Boundary value problem, three-point, shooting method. 
dealt with several analogues from the differential equations including both conjugate problems and right focal problems. For these questions in the context of dynamic equations on time scales, we mention the results by Bohner and Peterson [2], Chyan [3] and Henderson and Yin [10, 11].

Our uniqueness condition on solutions of (1) is in terms of a right disfocality condition, which we state in the form:

(C) Given points $a<p_{1}<p_{2}<b$, if $y$ and $z$ are solutions of (1) such that

$$
y\left(p_{1}\right)=z\left(p_{1}\right), y^{\prime}\left(p_{2}\right)=z^{\prime}\left(p_{2}\right),
$$

then $y(x)=z(x)$, for all $a<x<b$.

Remarks. (a) We notice that, if (C) is satisfied, then via an application of Rolle's Theorem, solutions of (1), (2) are unique when such solutions exist.

(b) Henderson [7] proved that hypotheses (A), (B) and (C) imply that solutions $y$ of (1) satisfying the right focal boundary conditions

$$
y\left(p_{1}\right)=y_{1}, y^{\prime}\left(p_{2}\right)=y_{2},
$$

$a<p_{1}<p_{2}<b$, and $y_{1}, y_{2} \in \mathbb{R}$, do in fact exist, and are unique by $(\mathrm{C})$.

In Section 2, we prove that solutions of (1), (2) depend continuously on boundary conditions. In Section 3, we make use of the continuous dependence result, the existence of solutions of (1), (3), and a Kamke-like result due to Vidossich [19] for solutions of (1), (3), to yield existence of solutions of (1), (2)). The paper is completed with a corollary illustrating that, if $f\left(x, v_{1}, v_{2}\right)$ satisfies certain monotonicity conditions with respect to $v_{1}$, then the crucial condition (C) is satisfied.

\section{Continuous Dependence}

In this section, we will show that solutions of (11), (2) depend continuously on boundary conditions.

Theorem 1. Assume that conditions (A), (B) and (C) hold. Given a solution $y(x)$ of (11) on $(a, b)$, an interval $[c, d] \subset(a, b)$, points $c<x_{1}<x_{2}<x_{3}<d$, and an $\epsilon>0$, there exists a $\delta(\epsilon,[c, d])>0$ such that, if $\left|x_{i}-t_{i}\right|<\delta, i=1,2,3$, and $c<t_{1}<t_{2}<t_{3}<d$, and if $\left|y\left(x_{1}\right)-z_{1}\right|<\delta$ and $\left|y\left(x_{3}\right)-y\left(x_{2}\right)-z_{2}\right|<\delta$, then there exists a solution $z(x)$ of (1) satisfying $z\left(t_{1}\right)=z_{1}, z\left(t_{3}\right)-z\left(t_{2}\right)=z_{2}$, and $\left|y^{(i)}(x)-z^{(i)}(x)\right|<\epsilon$ on $[c, d], i=0,1$.

Proof. Fix a point $p_{0} \in(a, b)$. Next define the set

$$
G=\left\{\left(x_{1}, x_{2}, x_{3}, C_{1}, C_{2}\right) \mid a<x_{1}<x_{2}<x_{3}<b, \text { and } C_{1}, C_{2} \in \mathbb{R}\right\} .
$$

$G$ is an open subset of $\mathbb{R}^{5}$. Next, define a mapping $\phi: G \rightarrow \mathbb{R}^{5}$ by

$$
\phi\left(x_{1}, x_{2}, x_{3}, C_{1}, C_{2}\right)=\left(x_{1}, x_{2}, x_{3}, u\left(x_{1}\right), u\left(x_{3}\right)-u\left(x_{2}\right)\right),
$$

where $u(x)$ is the solution of (1) satisfying the initial conditions $u\left(p_{0}\right)=C_{1}, u^{\prime}\left(p_{0}\right)$ $=C_{2}$. Condition (B) implies the continuity of solutions of initial value problems for (11) with respect to initial conditions, from whence we may derive the continuity of $\phi$. Moreover, condition (C) implies that $\phi$ is one-to-one. It follows from the Brouwer theorem on invariance of domain [18, page 199] that $\phi(G)$ is an open subset of $\mathbb{R}^{5}$ and that $\phi$ is a homeomorphism from $G$ to $\phi(G)$. The statement of the theorem is a direct result of the continuity of $\phi^{-1}$ and the fact that $\phi(G)$ is open. The proof is complete. 


\section{Existence of SOLUtions}

In this section, we employ the method of shooting to obtain solutions of (11), (2) under our uniqueness assumptions on (11), (3). Our arguments involve an application of a Kamke-like result due to Vidossich [19] as it applies to solutions of (1), (3).

Theorem 2. Assume that with respect to (11), conditions (A), (B), and (C) are satisfied. Given points $a<x_{1}<x_{2}<x_{3}<b$, and $y_{1}, y_{2} \in \mathbb{R}$, there exists a unique solution of (11), (2) on $(a, b)$.

Proof. Let $a<x_{1}<x_{2}<x_{3}<b$ and $y_{1}, y_{2} \in \mathbb{R}$ be selected. Then let $z(x)$ denote the solution of the initial value problem for (1) satisfying the initial conditions at $x_{1}$

$$
z\left(x_{1}\right)=y_{1}, \quad z^{\prime}\left(x_{1}\right)=0
$$

Next, define the set

$$
S=\left\{y\left(x_{3}\right)-y\left(x_{2}\right) \mid y(x) \text { is a solution of (11) satisfying } y\left(x_{1}\right)=z\left(x_{1}\right)\right\} .
$$

We observe first that $S$ is nonempty, since $z\left(x_{3}\right)-z\left(x_{2}\right) \in S$. In addition, by invoking Theorem 1 we conclude that $S$ is also an open subset of $\mathbb{R}$.

The remainder of the argument is devoted to showing that $S$ is also a closed subset of $\mathbb{R}$. To that end, we assume for the purpose of contradiction that $S$ is not closed. Then there exists an $r_{0} \in \bar{S} \backslash S$ and a strictly monotone sequence $\left\{r_{k}\right\} \subset S$ such that $\lim _{k \rightarrow \infty} r_{k}=r_{0}$.

We may assume, without loss of generality, that $r_{k} \uparrow r_{0}$. By the definition of $S$, we denote, for each $k \in \mathbb{N}$, by $u_{k}(x)$ the solution of (1) satisfying

$$
u_{k}\left(x_{1}\right)=z\left(x_{1}\right) \text { and } u_{k}\left(x_{3}\right)-u_{k}\left(x_{2}\right)=r_{k} \text {. }
$$

Then, for each $k \in \mathbb{N}$, there exists $t_{k} \in\left(x_{2}, x_{3}\right)$ such that

$$
u_{k}^{\prime}\left(t_{k}\right)=\frac{r_{k}}{x_{3}-x_{2}}
$$

so that

$$
u_{k}^{\prime}\left(t_{k}\right) \uparrow \frac{r_{0}}{x_{3}-x_{2}} \text { as } k \rightarrow \infty .
$$

Moreover, there exists a subsequence $\left\{k_{j}\right\} \subseteq\{k\}$ such that $\left\{t_{k_{j}}\right\}$ converges to some point $\tau_{0} \in\left[x_{2}, x_{3}\right]$. Of course,

$$
u_{k_{j}}^{\prime}\left(t_{k_{j}}\right) \uparrow \frac{r_{0}}{x_{3}-x_{2}} \text { as } j \rightarrow \infty .
$$

In conjunction with the existence of unique solutions of (11), (3), we apply the Kamke-like result for boundary value problems due to Vidossich [19] Corollary 1, page 3] to obtain

$$
\lim _{j \rightarrow \infty} u_{k_{j}}^{(i)}(x)=u^{(i)}(x)
$$

uniformly on compact subintervals of $(a, b), i=0,1$, where $u(x)$ is the solution of (11) satisfying the right focal boundary conditions

$$
u\left(x_{1}\right)=z\left(x_{1}\right), \quad u^{\prime}\left(\tau_{0}\right)=\frac{r_{0}}{x_{3}-x_{2}} .
$$


It follows in turn that

$$
u\left(x_{3}\right)-u\left(x_{2}\right)=\lim _{j \rightarrow \infty}\left[u_{k_{j}}\left(x_{3}\right)-u_{k_{j}}\left(x_{2}\right)\right]=r_{0},
$$

or that $r_{0} \in S$, a contradiction. Thus, $S$ is a closed subset of $\mathbb{R}$.

In summary, $S$ is a nonempty subset of $\mathbb{R}$ that is both open and closed. We have $S \equiv \mathbb{R}$, and so by choosing $y_{2} \in S$, there is a corresponding solution $y(x)$ of (1) such that

$$
y\left(x_{1}\right)=y_{1}, \quad y\left(x_{3}\right)-y\left(x_{2}\right)=y_{2} .
$$

This completes the proof.

We conclude the paper with a corollary exhibiting sufficient conditions to fulfill the uniqueness condition $(\mathrm{C})$.

Corollary 1. Assume that conditions (A) and (B) hold, and in addition assume that for each fixed $\left(x, v_{2}\right)$, the function $f\left(x, v_{1}, v_{2}\right)$ is strictly increasing as a function of $v_{1}$. Then condition (C) holds, and hence there exist unique solutions of (10), (2)).

Proof. Assume the conclusion concerning condition (C) is false. Then there are distinct solutions $y$ and $z$ of (11) and points $a<p_{1}<p_{2}<b$ so that

$$
y\left(p_{1}\right)=z\left(p_{1}\right), \quad y^{\prime}\left(p_{2}\right)=z^{\prime}\left(p_{2}\right) .
$$

By (B), $y^{\prime}\left(p_{1}\right) \neq z^{\prime}\left(p_{1}\right)$, and so we may assume with no loss of generality that $y^{\prime}\left(p_{1}\right)>z^{\prime}\left(p_{1}\right)$. Set $w=y-z$. Then

$$
w\left(p_{1}\right)=0=w^{\prime}\left(p_{2}\right) \text { and } w^{\prime}\left(p_{1}\right)>0 .
$$

We may also assume that $p_{2}$ is the first point greater than $p_{1}$ for which $w^{\prime}$ vanishes. Then,

$$
w\left(p_{2}\right)>0, w^{\prime}\left(p_{2}\right)=0, \text { and } w^{\prime \prime}\left(p_{2}\right) \leq 0 .
$$

However, by the strict monotonicity growth in $f\left(x, v_{1}, v_{2}\right)$ with respect to $v_{1}$,

$$
\begin{aligned}
w^{\prime \prime}\left(p_{2}\right) & =y^{\prime \prime}\left(p_{2}\right)-z^{\prime \prime}\left(p_{2}\right) \\
& =f\left(p_{2}, y\left(p_{2}\right), y^{\prime}\left(p_{2}\right)\right)-f\left(p_{2}, z\left(p_{2}\right), z^{\prime}\left(p_{2}\right)\right) \\
& =f\left(p_{2}, y\left(p_{2}\right), z^{\prime}\left(p_{2}\right)\right)-f\left(p_{2}, z\left(p_{2}\right), z^{\prime}\left(p_{2}\right)\right) \\
& >0,
\end{aligned}
$$

which is a contradiction. The proof is complete.

\section{ACKNOWLEDGMENT}

This work was undertaken while the first Author was visiting the University of New South Wales. The third Author gratefully acknowledges the financial support of UNSW.

\section{REFERENCES}

[1] C. Bai and J. Fang, Existence of multiple positive solutions for nonlinear $m$-point boundary value problems, J. Math. Anal. Appl. 281 (2003), 76-85. MR1980075 (2004b:34035)

[2] M. Bohner and A.C. Peterson, Dynamic Equations on Time Scales, An Introduction with Applications, Birkhäuser, Boston, 2001. MR1843232 (2002c:34002)

[3] C.J. Chyan, Uniqueness implies existence on time scales, J. Math. Anal. Appl. 258 (2001), 359-365. MR 1828110 (2002e:34004)

[4] J.M. Davis and J. Henderson, Uniqueness implies existence for fourth-order Lidstone boundary value problems, Panamer. Math. J. 8 (1998), 23-35. MR.1657450 (99i:34033) 
[5] C.P. Gupta and S.I. Trofimchuk, Solvability of a multi-point boundary value problem and related a priori estimates, Canad. Appl. Math. Quart. 6 (1998), 45-60. MR 1638415 (99f:34020)

[6] P. Hartman, On $N$-parameter families and interpolation problems for nonlinear ordinary differential equations, Trans. Amer. Math. Soc. 154 (1971), 201-226. MR0301277|(46:435)

[7] J. Henderson, Existence of solutions of right focal point boundary value problems for ordinary differential equations, Nonlin. Anal. 5 (1981), 989-1002. MR0633013 (82j:34015)

[8] J. Henderson, Existence theorems for boundary value problems for $n$ th-order nonlinear difference equations, SIAM J. Math. Anal. 20 (1989), 468-478. MR0982673 (90a:39003)

[9] J. Henderson, Focal boundary value problems for nonlinear difference equations, I, II, $J$. Math. Anal. Appl. 141 (1989), 559-567, 568-579. MR1009063 (90g:39003)

[10] J. Henderson and W.K.C. Yin, Existence of solutions for fourth order boundary value problems on a time scale, J. Differ. Eqs. Appl. 9 (2003), 15-28. MR1958300 (2003k:34041)

[11] J. Henderson and W.K.C. Yin, Two-point and three-point problems for fourth order dynamic equations, Dynam. Systems Appl. 12 (2003), 159-169. MR1989029|(2004d:34034)

[12] L.K. Jackson and K. Schrader, Existence and uniqueness of solutions of boundary value problems for third order differential equations, J. Diff. Eqs. 9 (1971), 46-54. MR0269920 (42:4813)

[13] G. Klaasen, Existence theorems for boundary value problems of $n$th order ordinary differential equations, Rocky Mtn. J. Math. 3 (1973), 457-472. MR0357944 (50:10409)

[14] A. Lasota and M. Luczynski, A note on the uniqueness of two point boundary value problems I, Zeszyty Naukowe UJ, Prace matematyezne 12 (1968), 27-29. MF0224900 (37:499)

[15] Ruyun Ma, Existence theorems for a second-order three-point boundary value problem, $J$. Math. Anal. Appl. 212 (1997), 430-442. MR1464888 (98h:34041)

[16] Ruyun Ma, Existence and uniqueness of solutions to first-order three-point boundary value problems, Appl. Math. Lett. 15 (2002), 211-216. MR1880760 (2002k:34032)

[17] A.C. Peterson, Existence-uniqueness for focal-point boundary value problems, SIAM J. Math. Anal. 12 (1981), 173-185. MR0605428 (83h:34017)

[18] E. H. Spanier, Algebraic Topology, McGraw-Hill, New York, 1966. MR.0210112 (35:1007)

[19] G. Vidossich, On the continuous dependence of solutions of boundary value problems for ordinary differential equations, J. Diff. Eqs. 82 (1989), 1-14. MR1023298 (90j:34036)

[20] B. Yang, Boundary Value Problems for Ordinary Differential Equations, Ph.D. Dissertation, Mississippi State University, Mississippi State, MS, 2002.

Department of Mathematics, Baylor University, Waco, Texas 76798-7328

E-mail address: Johnny_Henderson@baylor.edu

Department of Mathematics, Baylor University, Waco, Texas 76798-7328

E-mail address: Basant_Karna@baylor.edu

Current address: Department of Mathematics, Marshall University, Huntington, West Virginia 25755-2560

E-mail address: karna@marshall.edu

School of Mathematics, The University of New South Wales, Sydney 2052, Australia

E-mail address: cct@maths.unsw.edu.au 